l-glutaminsäure eine besondere Wirkung bei $E$. coli, S. haemolyticus und D. pneumoniae ab.

Danach scheint die überragende Wirkung der p-Amino-benzoyl-l-glutaminsäure als Wuchsstoff nur für ganz bestimmte Stämme von Streptobacterium plant. zu gelten. Diese sind außerdem - gemäß einer mündlichen Mitteilung von E.F.M öller, Heidelberg - in ihren Eigenschaften besonders . variabel. Eine allgemeine Bedeutung als Wirkungsgruppe eines Wachstumsfaktors kommt der p-Aminobenzoyl-l-glutaminsäure wohl nicht zu. Sollten sich die Angaben vón Auhagen reproduzieren lassen, dann würde sich daraus ergeben, daß zwar der Wuchsstoff für verschiedene Bakterienarten und-stämme der gleiche, nämlich p-Amino-benzoesäure, aber die Verknüpfung mit dem Träger eine verschiedene ist. Es ist naheliegend, anzunehmen, daß außer Glutaminsäure auch andere Aminosäuren als Bindeglieder auftreten.

p-Nitro-benzolsulfonyl-l-glutaminsäure (IV): Aus $l(+)$-Glutaminsäure und $p$-Nitro-benzolsulfochlorid in $n / 1-\mathrm{NaOH}$ bei $50-80^{\circ}$. Schwach gelbstichige Nadeln vom Schmp. $186^{\circ}$ (unkorr.; aus $\mathrm{H}_{2} \mathrm{O}$ umkrystallisiert). $[\alpha]_{D}$ $=-22,8^{\circ}$ (7,9-proz. Lsg. in 1,2-n. NaOH).

Saures Kaliumsalz: Schwach gelbliche, lichtempfindliche Krystalle vom Zers.-Pkt. $247^{\circ}$ (unkorr.; aus $\mathrm{H}_{2} \mathrm{O}$ umkryst.). Löslich in verdünnter Lauge, warmem Eisessig, unlöslich in heißem Alkohol.

p-Aminobenzolsulfonyl-glutaminsäure (II) entstand aus IV durch katalytische Hydrierung mit PalladiumTierkohle in Eisessig. Aus Wasser schwach bräunlichgelbe Krystalle vom Zers.-Pkt. 227-2310 (unkorr.).

Bei der Durchführung der bakteriologischen Versuche wurden wir von Frl. E. Schreiber aufs beste unterstiutzt.

\title{
Frequenzanalyse bioelektrischer Potentialschwankungen ${ }^{1}$
}

\author{
Von Bruno Franek
}

Aus der neurophysiologischen Abt. der Universitäts-Nervenklinik, Wien

(Z. Naturforschg. 1, 231-236 [1946]; eingegangen am 18. März 1946)

\begin{abstract}
Mit der Frage nach dem Entstehungsort der gefilterten polyfrequenten bioelektrischen Potentialschwankungen wurde das Verhältnis einer jeden Frequenz zu allen anderen Frequenzen an den verschiedenen Ableitepunkten untersucht-Die verschiedenen Kopfableitepunkte waren dabei so ausgesucht, daß sie sich etwa mitten über cytoarchitektonisch stark differierenden Feldern der Großhirnrinde befanden. Es wurden von einem prä- und einem postzentralen Punkte höherfrequente Hirnaktionsströme abgeleitet, die sich durch plötzlichen Beginn, synchronen und abklingenden Schwingungsverlauf sowie durch eine enorme Spannungsüberhöhung und klare ortsbedingte Differenzierung auszeichnen. Sie dürften den funktionellen Ausdruck der darunter befindlichen morphologischen Unterschiedlichkeit der Großhirnrinde darstellen. Der enkephalogene Charakter der höheren Frequenzen des passiven EEG wird sehr in Frage gestellt, da sich an vielen Stellen des Körpers die prinzipiell gleichen polyfrequenten Erscheinungen mit parallel verlaufender Spannungsverminderung und gewissen Beziehungen zum Blutkreislaufsystem nachweisen lassen. Dagegen wird durch Frequenzanalyse von dicht nebeneinander abgeleiteten Hirnaktionsströmen die bioelektrische Differenzierung der Großhirnrinde bestätigt.
\end{abstract}

$\mathrm{E}$ sind im Laufe der letzten zwanzig Jahre eine große Zahl der verschiedensten bioelektrischen Erscheinungsformen bei direkter Hirnableitung, wie bei indirekter Ableitung von der Kopfhaut des Menschen bekannt geworden. Über die Ätiologie dieser elektrischen Funktionen aber ist man noch zu keiner allgemein verbindlichen Auffassung gekommen. Wir glauben einen nicht unwichtigen

1 Kurze Zusammenfassung einer Arbeit im Rahmen des The od or - M ey nert-Stipendiums, das mir von der Wiener Medizinischen Gesellschaft in großzügiger Weise gewährt wurde.
Hinderungsgrund für den EEG ${ }^{2}$-Fortschritt in der methodischen Einseitigkeit zu erblicken, daß fast alle EEG-Forschung seit :E. Berger ${ }^{3}$ eine ausschließliche Erforschung der stark in den Vordergrund tretenden $\alpha$-Wellen darstellte. Selbst die Betrachtung aller denkbar feinsten Nuancen dieser $\alpha$-Wellen aber dürfte nicht imstande sein, uns ein auch nur annähernd adäquates Bild vom komplexen elektrofunktionellen Hirngeschehen zu vermitteln.

2 EEG = Elektrenkephalographie.

3 Arch. Psychiatrie [1929-1938]. 
Dagegen entfaltet sich aber der im bisher üblichen ungefilterten EEG unansehnliche Bereich der höherfrequenten Potentialschwankungen, der mit dem stiefmütterlichen Terminus , $\beta$-Wellen“*) bedacht wurde, bei genauerer Betrachtung zu einem weiten Feld, das wegen der Vielfalt seiner wohldifferenzierten Erscheinungen entsprechend mehr Angriffsmöglichkeiten zur Erforschung bieten sollte ${ }^{4}$. So wurden also alle Potentialschwankungen bis zu einer Frequenz von $150 \mathrm{~Hz}$ systematisch untersucht.

\section{Methodik}

Es wurde ein elektrisches Filtergerät benützt, wie es von J.Prast angegeben wurde ${ }^{5}$. Die hier angewendeten speziellen Siebketten wurden von Hrn. Ing. H. M a r k o ausgearbeitet, dem ich besonders für seine tatkräftige Anteilnahme an dieser Arbeit danke.

\section{Registriergerät}

Zur Registrierung wurden Oscillographenschleifen benützt. Die Frequenzcharakteristik des Gerätes einschließlich Oscillographenschleifen (Siemens Type EKG) geht bis etwa $800 \mathrm{~Hz}$, was für die vorliegenden Probleme vollkommen ausreichend ist. Die Stromversorgung des Gerätes erfolgt vollständig aus Akkumulatorenbatterien, so daß eine außerordentlich hohe Konstanz und Störungsfreiheit erreicht wird. Verstärker und Batterie sind gegen die Umgebung vollkommen metallisch abgeschirmt, wodurch die Einwirkung äußerer Störfelder weitgehend vermieden wird. Der mechanische Aufbau des Verstärkers ist so durchgeführt, daß durch federnde Aufhängung der empfindlichen Schaltelemente, insbesondere der ersten Stufe, auch mechanische Erschütterungen keinen wesentlichen Einfluß auf die Anzeige der Oscillographenschleife haben können.Verstärker 'und Oscillograph sind in verschiedenen Räumen untergebracht, so daß sowohl elektrische, wie auch mechanische Störungen, die durch den Oscillographenmotor entstehen könnten, keine Wirkung auf den Verstärker haben.

\section{Elektroden}

Zur Verbesserung der Ableitebedingungen wurden Kleinelektroden mit einer Ableitefläche von etwa $4 \mathrm{~mm}^{2}$

* Zur Frage der Bestimmung des „, $\beta$-Wellen“-Begriffes hat A. E: Kornmüller neuerdings in seinem Buche „Klinische Elektrenkephalographie“ den sinnvoll vermittelnden Vorschlag gemacht, neben der ausdrücklichen Bezeichnung jeder höheren Frequenz aus historischen Rücksichten den Begriff „, $\beta$-Wellen“ wohl noch beizubehalten, ihn jedoch auf Schwingungen einer Frequenz von $20 \mathrm{~Hz}$ zu beschränken.

${ }^{4}$ Dagegen R. Jung, Nervenarzt 12, 570 [1939].

5 J.Prast u. B. Franek (erscheint demnächst in dieser Z., dort auch ausführlichere Darstellung der Methodik). verwendet. Zum Halten dieser sehr kleinen Elektroden ließ ich mir Fassungen bauen, die wie kleine Hütchen über die Ableitungsstellen aufgesetzt werden und eine Höhe von etwa $3,5 \mathrm{~cm}$, sowie einen Kreisdurchmesser dort, wo sie dem Kopfe anliegen, von 3,5 cm haben. Nach oben hin ist durch die Fassung ein enger Kanal gebohrt, in den der Elektrodenzylinder von unten eingeschoben werden kann und durch eine kleine Spiralfeder so gehalten wird, daß er mit konstantem Druck der Ableitungsstelle anliegt. Durch den Elektrodenzylinder wird ein dünner Wollfaden gezogen, der von oben mit physiologischer Kochsalzlösung getränkt werden kann, ohnedaß irgend etwas an der Halteyorrichtung verändert zu werden braucht. Die Elektrodenfassung ist so ausgeschliffen, daß sie sich der Kopfwölbung gut anschmiegt und gleichzeitig dabei die Haare auseinanderhält. — Sie besteht aus Trolitul, einem Material, welches ganz hervorragend für solche Zwecke geeignet ist; es isoliert in denkbar hohem Grade und hat zudem die vorteilhafte Eigenschaft, im geschliffenen Zustande etwa so durchsichtig wie Glas zu sein, was die Möglichkeit einer Kontrolle gibt, ob die Kleinelektrode nun auch an genau der gewünschten Stelle sitzt. Das spezifische Gewicht dieses Kunststoffes ist so gering, daß die ganze Elektrodenfassung nur ein Gewicht von etwa $25 \mathrm{~g}$ hat. Mit Hilfe eines durchlöcherten Gummihaltebandes können mehrere solcher Kleinelektrodenfassungen bequem über den gewünschten Ableitepunkten befestigt werden.

\section{Versuchsbedingungen}

Es wurde, um Fehldeutungen der selektiv gefilterten Kurven vorzubeugen, in jedem Falle vom gleichen $\mathrm{Ab}$ leitepunkt die normale frequenzunbeschränkte Kurve mit aufgenommeń; außerdem wurde jedesmal eine Kurve mit extremer Beschränkung der höheren Frequenzen dazu aufgezeichnet. Eine solche Anordnung läßt uns bei der nötigen Erfahrung alle technischen Störungen, eingestreute Herzaktionsströme und selbst sehr schwache Aktionsströme der Kopfmuskeln mit Sicherheit erkennen und so den Filterbefund vor Fehldeutungen schützen.

Bei den noch näher zu beschreibenden Hirnaktionsströmen wurde gleichzeitig von zwei Punkten abgeleitet. Der eine Ableitepunkt befand sich dabei über dem mittleren Teil des Gyrus centralis anterior, der andere über dem unteren Teil des Gyrus centralis posterior.

Zur Ableitung der höherfrequenten Schwingungen des sogenannten passiven EEG ${ }^{6}$ suchten wir solche Stellen an der Kopfhaut aus, die über cytoarchitektonisch stark differierenden Feldern der Großhirnrinde lagen. Ich stützte mich dabei auf Arbeiten von C.v. E c o n o m o ${ }^{7}$, dessen Felderbezeichnungen ich auch verwende, wenn ich die Ableitestellen an der Kopfhaut durch die darunter

${ }^{6}$ H. R o hr a ch e r, Die elektr. Vorgänge im menschl. Gehirn, Leipzig 1942.

7 C. v. Economo u. Koskinas, Cytoarchitekton. Atlas der Großhirnrinde des erwachsenen Menschen, Springer Berlin 1929. Ich konnte ferner myeloarchitektonische Arbeiten seines wissenschaftlichen Nachlasses benützen, den mir seine Gattin, Baronin v. Economo, in liebenswürdiger Weise zur Verfügung gestellt hat. Eine Zusammenfassung dieser Arbeiten beabsichtigen wir bald zu veröffentlichen. 
sich im engen Umkreis befindenden cystoarchitektonischen Felder folgendermaßen charakterisiere:

$$
\begin{gathered}
\text { P(unkt) 1: } \mathrm{O}_{\mathrm{B}}, \mathrm{O}_{\mathrm{C}}, \text { P 2: } \mathrm{P}_{\mathrm{B}}, \mathrm{P}_{\mathrm{C}} \cdot \text { P 3: } \mathrm{F}_{\mathrm{A} \gamma} \\
\text { P 4: } \mathrm{F}_{\mathrm{Dm}}, \mathrm{F}_{\mathrm{D} \Delta} \text { P 5: } \mathrm{T}_{\mathrm{B}, \mathrm{C}, \mathrm{D}}, \mathrm{J}_{\mathrm{B}} \cdot \\
\text { P 6: } \mathrm{T}_{\mathrm{El}}, \mathrm{P}_{\mathrm{H}_{\mathrm{p}}}, \mathrm{P}_{\mathrm{Fem}}, \mathrm{P}_{\mathrm{G}}, \mathrm{P}_{\mathrm{H}} \cdot
\end{gathered}
$$

\section{Hirnaktionsströme}

Bei Versuchen im Jahre 1942 am Kaiser-Wilhelm-Institut für Hirnforschung gelang bereits die

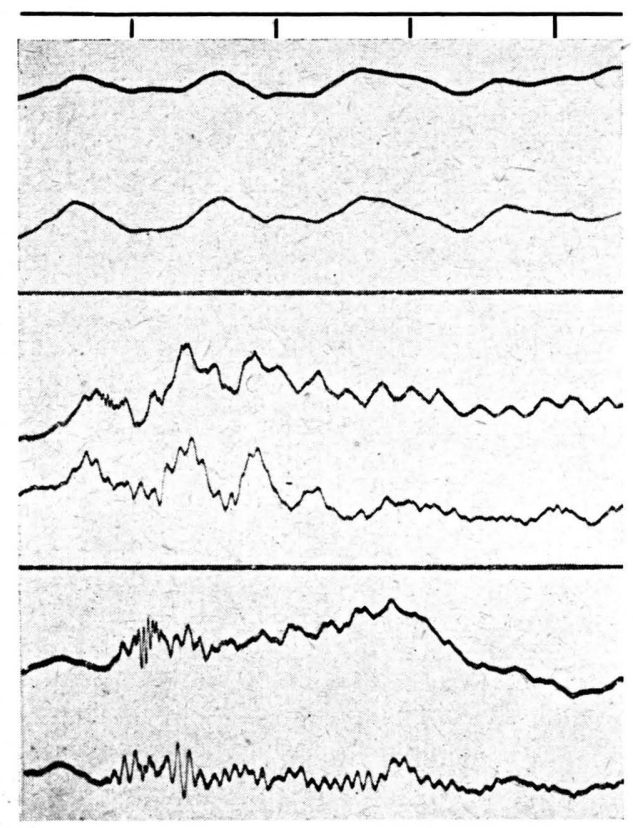

Abb. 1. Im oberen Drittel „Ruheströme“ bei Ableitung von einem postzentralen (obere Kurve) und einem präzentralen Punkte (untere Kurve). Die beiden Kurven des mittleren Drittels der Abbildung stellen den unmittelbar darauf folgenden Hirnaktionsstrom dar und die des unteren Drittels einen weiteren, bald darauf folgenden. Zeitmarke $=1 / 10$ Sek.

Ableitung gefilterter eindeutiger Hirnaktionsströme von einer eng umschriebenen occipitalen Region, genau zeitlich bedingt durch die Augenbelichtung der Versuchsperson ${ }^{8}$. Jetzt ist es sogar möglich, das frequenzanalytische Verhalten von Hirnaktionsströmen zu zeigen, welche gleichzeitig von prä- und postzentralen Ableitepunkten aufgenommen wurden, die nicht weiter als $1,7 \mathrm{~cm}$ voneinander entfernt waren.

8 B. Franek, Über eine Frequenzanalyse des Elektrenkephalogramms. Dissertation Berlin 1944.
Ein wichtiges Kennzeichen der Hirnaktionsströme bildet das mit einer starken vorausgehenden $\alpha$-Reduktion ${ }^{9}$ verbundene plötzliche Auftreten einer höherfrequenten Schwingungsgruppe. Man hat den Eindruck, daß es sich da um einen kurzen kräftigen Impuls handeln muß, der eine augenblickliche starke Potentialdifferenz bedingt, die sich mit der Tendenz des Spannungsausgleiches bald wieder zum gewohnten normalen passiven EEG ausschwingt.

Ein weiteres bedeutsames Merkmal ergibt sich dadurch, daß neben dem gleichzeitigen Auftreten gleicher Frequenzen an beiden Ableitepunkten, im beschriebenen Falle (Abb.1) der $20-\mathrm{Hz}-$ Schwingungen, auch ein gleichzeitiges sehr deutliches Auftreten ganz verschiedener Frequenzen sich beobachten läßt: hier z. B. präzentral $120 \mathrm{~Hz}$, postzentral $42 \mathrm{~Hz}$, von einer $360-\mathrm{Hz}$ Schwingung überlagert. Der synchrone, eruptive Beginn beider Hirnaktionsströme läßt auf ein gemeinsames Agens schließen, das sich bei relativ dicht beieinander liegenden Ableitestellen der Kopfhaut durch eindeutig unterschiedliche bioelektrische Effekte ausdrückt.

Das wichtigste Kriterium aber ist die enorme Spannungsüberhöhung aller beteiligten Frequenzkomponenten, die sich sinnfällig aus dem

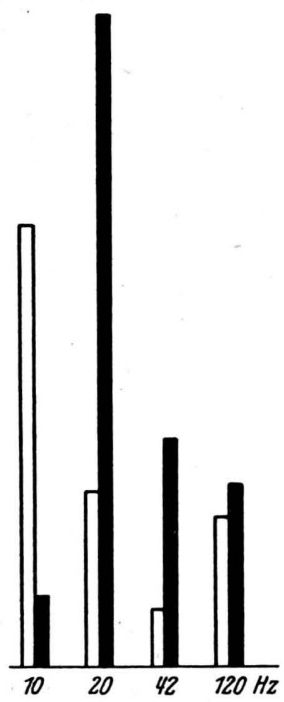

Abb. 2. Spannungsüberhöhung der höherfrequenten Komponenten des Hirnaktionsstroms (weiß) imVergleich zu den entsprechenden passiven EEG (schwarz). Vergleich mit den Spannungsintensitäten der entsprechenden Frequenzkomponenten des passiven EEG ergibt (Abb.2). Derartige Hirnaktionsströme - in unserem Falle bei wiederholtem Bewegen des Oberschenkels - ihr synchrones und gleichzeitig frequenzdifferenziertes Verhalten, die Spannungsüberhöhung ihrer Frequenzkomponenten, und schließlich ihre klare ortsbedingte Unterschiedlichkeit dürften nichts anderes darstellen, als den funktionellen Ausdruck der darunter sich befindenden morphologischen Unterschiedlichkeit der Großhirnrinde.

${ }^{9}$ H. H. J a spers u. R. M. Cruikshank, J. gen. Psychol. 17, 29 [1937]. 


$$
\text { Passives EEG }
$$

Die Potentialschwankungen der verschiedenen Frequenzbereiche erfolgen nicht im kontinuierlichen Spannungsverlauf, sondern in für den jeweiligen Frequenzbereich charakteristischen Spannungsstößen, die von kurzen Intervallen einer geringeren Spannungsproduktion unterbrochen werden. Das auffallendste Merkmal der verschiedenen Frequenzkomponenten des passiven EEG ist die charakteristische Eigenständigkeit in qualitativer Hinsicht, wie sie aus Tabelle 1 ungefähr erkennbar ist.

Da bei einem gleichzeitigen Vergleich der verschiedenen Frequenzen untereinander jeder Synchronismus vermißt wird, dürfen wir wohl mit einer Vielzahl verschiedener spannungserzeugen-

\begin{tabular}{|c|c|c|c|}
\hline $\mathrm{Hz}$ & $\begin{array}{c}\text { Frequenz- } \\
\text { stabilität }\end{array}$ & $\begin{array}{c}\text { Ampli- } \\
\text { tuden- } \\
\text { stabilität }\end{array}$ & Besonderheiten \\
\hline 10 & -++ & -+ & $\begin{array}{l}\text { ausgeprägte regionäre } \\
\text { Unterschiede }\end{array}$ \\
\hline 18 & - & -- & $\begin{array}{l}\text { charakteristische eruptive } \\
\text { Gruppenbildung }\end{array}$ \\
\hline 20 & --+ & -+ & $\begin{array}{l}\text { der 10-Hz-Schwingung ent- } \\
\text { gegengesetzte deutliche } \\
\text { regionäre Unterschiede }\end{array}$ \\
\hline 22 & -+ & -- & $\begin{array}{l}\text { wie bei } 18 \mathrm{~Hz} \text {; aber öfter } \\
\text { und deutlicher }\end{array}$ \\
\hline$\sim 25$ & --- & --- & indifferentes Kurvenbild \\
\hline 27 & -+ & -+ & $\begin{array}{l}\text { verhält sich ganz ähnlich } \\
\quad \text { wie } 22 \mathrm{~Hz}\end{array}$ \\
\hline 30 & -++ & -+ & $\begin{array}{l}\text { regionäre Unterschiede } \\
\text { (Oberwelle von } 10 \mathrm{~Hz} \text { ) }\end{array}$ \\
\hline$\sim 33$ & $-\cdots$ & -- & sehr unregelmäßig \\
\hline 40 & --+ & -+ & $\begin{array}{l}\text { regionäre Unterschiede } \\
\quad \text { (Oberwelle von } 20 \mathrm{~Hz} \text { ) }\end{array}$ \\
\hline 42 & --+ & -+ & $\begin{array}{l}\text { starke und häufige eruptive } \\
\text { Gruppenbildung }\end{array}$ \\
\hline 47 . & -+ & -+ & ganz ähnlich wie $42 \mathrm{~Hz}$ \\
\hline 52 & -+ & -+ & $\begin{array}{l}\text { ähnlich wie } 42 \mathrm{~Hz} \text {, noch } \\
\text { häufiger auftretend }\end{array}$ \\
\hline 57 & --+ & -+ & wie $52 \mathrm{~Hz}$ \\
\hline$\sim 73$ & --- & $-\ldots$ & vollkommen unregelmäßig \\
\hline 82 & +++ & ++ & $\begin{array}{l}\text { stark ausgeglichenes Kur- } \\
\text { venbild }\end{array}$ \\
\hline$\sim 95$ & -- & -- & indifferent \\
\hline 107 & ++++ & +++ & $\begin{array}{l}\text { vollkommen beständig } \\
\text { Spindelbildung }\end{array}$ \\
\hline 120 & +++ & ++ & weitgehend konstant. \\
\hline
\end{tabular}

Tab. 1. Allgemeine Charakteristik der selektiv gefilterten Frequenzen. der Elemente rechnen, die in andauernder Tätigkeit sind, und die in einer für sie jeweils charakteristischen Eigenart Potentialschwankungen einer bestimmten Frequenz, einer bestimmten Intensität und rhythmischen Gruppenbildung erzeugen (vergleiche z. B. Abb. 3).

Wir haben bei unipolarer Ableitung ${ }^{10}$ an allen Punkten der Kopfhaut in quantitativer, wie in qualitativer Hinsicht - mit Ausnahme der $\alpha$ - und $\beta$-Wellen nebst deren Oberwellen - überall ein gleichförmiges frequenzanalytisches Bild ohne

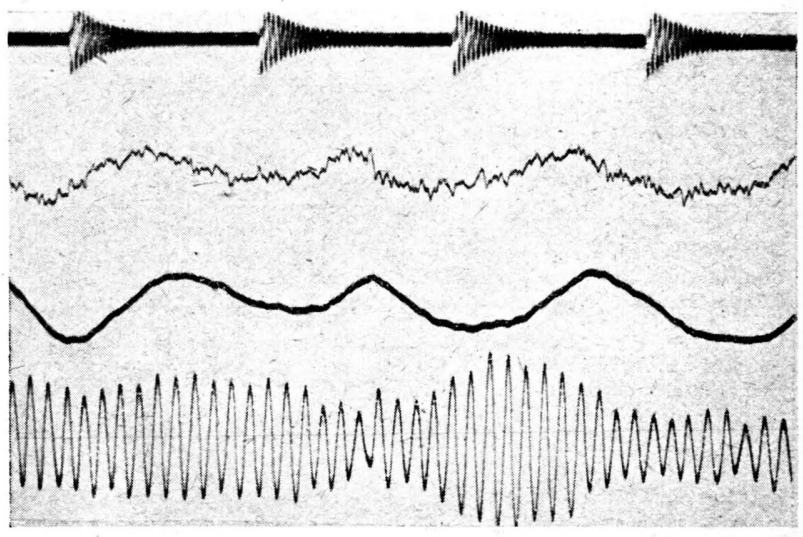

Abb. 3. Oscillogramm einer unipolaren Ableitung von einem temporalen Punkte. Die untere Kurve zeigt bei $112 \mathrm{~Hz}$ resonierender Einstellung des Filters die sehr konstanten $107 \mathrm{~Hz}$-Schwingungen mit den charakteristischen Spindelbildungen. Die mittlere Kurve zeigt das bisher übliche normale EEG mit den lediglich stark hervortretenden $\alpha$-Schwingungen. Die obere Kurve zeigt die frequenzunbeschränkte Kontrollregistrierung, die alle etwaigen Stör- oder Muskelaktionsströme kenntlich macht. Ganz oben die Zeitmarke (1/10 Sek.).

jeden regionären Unterschied. Im Gegensatz zum Hirnaktionsstrom ist also bei den Bedingungen des passiven EEG eine brauchbare bioelektrische örtliche Differenzierung nicht gegeben.

\section{Spektrum des übrigen Körpers}

Um zu ergründen, ob etwa doch nur die relativ geringfügige morphologische Unterschiedlichkeit innerhalb der Großhirnrindenstruktur es ist, die keine brauchbare bioelektrische Lokalisierbarkeit zuließe, wurde über den Wirbeldornfortsätzen einer Patientin abgeleitet, die an einer vollkommenen Querschnittslähmung erkrankt war. Unterhalb $D_{5}$ hatte sie vollkommenen Sensibilitätsverlust; jede

10 A. E. Kornmüller u. R. Janzen, Arch. Psychiatrie 109, 261 [1939]. 
Motorik war in dem betreffenden Gebiet erloschen. Aber auch in diesem Falle wurde festgestellt, daß bei engem Abgriff der Ableitungen (zwischen $\mathrm{D}_{4}$ und $\mathrm{D}_{6}$ ), wie auch bei weit auseinanderliegenden (zwischen $\mathrm{D}_{2}$ und $\mathrm{D}_{10}$ ) keine besonderen Anderungen innerhalb der höheren Frequenzen an den verschiedenen Ableitepunkten wahrzunehmen waren, und daß schließlich an allen Ableitepunkten des übrigen Körpers prinzipiell die gleichen Frequenzen in der beschriebenen Eigenart abgeleitet werden konnten.

In einer weiteren Untersuchung bei der Patientin mit der vollkommenen Querschnittslähmung wurde folgende Elektrodenanordnung getroffen: Es wurde am li. Unterschenkel $5 \mathrm{~cm}$ oberhalb des

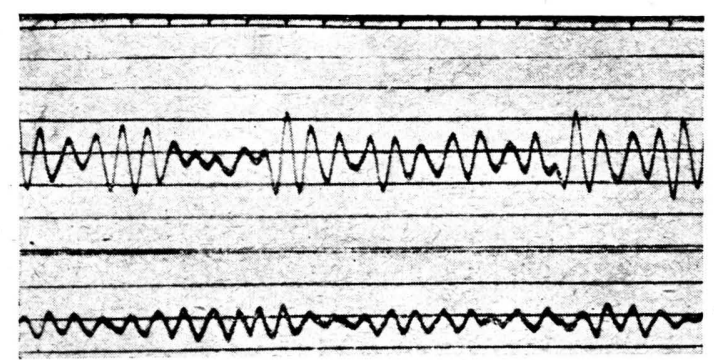

Abb. 4. $\alpha$-Welle bei bipolarer Ableitung vom Unterschenkel. Die untere Kurve zeigt die beträchtliche $\alpha$-Wellenreduktion 2 Min. nach Unterbindung der Blutzufuhr des betreffenden Gebietes. Zeitmarke ${ }^{1 / 10}$ Sek.

li. Knöchels die eine, $10 \mathrm{~cm}$ kniewärts von dieser die andere Elektrode angelegt. (Hierbei handelte es sich um die gebräuchlichen Elektroden mit einer Ableitefläche von etwa 1,0 cm Durchmesser.) Alle von einem Punkte oberhalb der Ableitung durch die Körperoberfläche kommenden Ströme dürften bei unseren mit den Stromlinien in gleicher Richtung befindlichen hintereinander liegenden Elektroden keine Potentialdifferenz, d. h. auch keine registrierbaren Schwingungen erzeugen können. Gefilterte Muskelaktionsströme aus der unmittelbaren Umgebung waren durch die Ausdehnung der motorischen Lähmung ausgeschlossen, durch Nerven in das subcutane Gewebe fortgeleitete Nervenaktionsströme desgleichen; Muskelaktionsstromeinflüsse aus den gesunden oberen Teilen des Körpers verboten sich ebenfalls aus der Anordnung unserer Elektroden. Das einzige, was noch an funktionierender ausgedehnter Substanz vorlag, war in etwa noch der trophisch nervöse Apparat, in ganz geringer Weise die Tiefensensi- bilität sowie das Blutkreislaufsystem. Unter diesen Bedingungen setzten wir nun unterhalb des li. Kniegelenkes einen zirkulären Druck von $260 \mathrm{~mm} / \mathrm{Hg}$.

Schon nach kurzer Zeit des Druckes nahmen die Amplituden aller Frequenzen deutlich an Größe ab. Die $\alpha$-Wellenreduktion nach einer solchen Blutstauung von etwa 2 Min. sehen wir deutlich in der Abb. 4. - Es war bei allen angestellten Versuchen eine ausgesprochene Amplitudenreduktion festzustellen, und zwar bis zu $50 \%$. Bei Freigabe des Blutkreislaufes durch Wegnahme des zirkulären Druckes gewannen dann die verschiedenen Frequenzen bald wieder ihre ursprüngliche Amplitudengröße zurück.

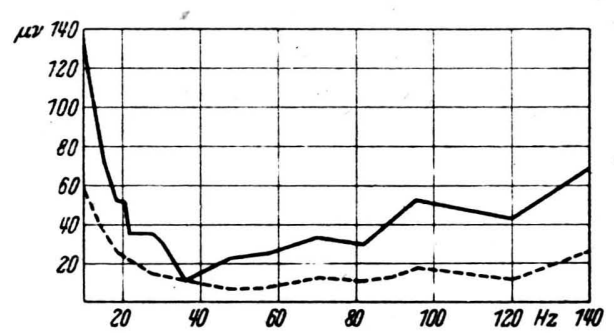

Abb. 5. Intensitätsvergleich der Spannungen des gesamten Frequenzspektrums bei Ableitung von einem occ. Punkte (obere Kurve) mit denen bei Ableitung vom Unterschenkel (gestrichelte Kurve). Die Spannungsintensität der einzelnen Frequenzen wurde aus deren durchschnittlicher Amplitudengröße und dem jeweiligen Verstärkungskoeffizienten ermittelt.

Sehr aufschlußreich ist ferner der Intensitätsvergleich der Spannungen der jeweiligen Frequenzen an verschiedenen Ableitestellen der Körperoberfläche. Die obere Kurve der Abb. 5 zeigt die Spannungen aller gemessenen Frequenzen bei $\mathrm{Ab}$ leitung von einem occipitalen Punkt, die untere bei einer Ableitung von den Punkten des li. Unterschenkels, die beim letzten Versuch genauer definiert wurden. Die Werte wurden durch Messung der durchschnittlichen Amplitudenhöhe der jeweilichen Frequenzen ermittelt.

\section{Diskussion}

Wahrscheinlich steht also, wie unsere Versuche sinnfällig zeigen, das Blutkreislaufsystem in einer gewissen Beziehung zum Auftreten der polyfrequenten Erscheinungen an der Körperoberfläche. Es fragt sich nur, ob diese Beziehung eine nur beiläufige, oder sogar eine ursächliche sei. Beide Möglichkeiten stehen offen und schließen sich durch 
unsere bisherigen Versuche noch nicht gegenseitig aus.

Wenn es uns auch wegen der Unkenntnis der spannungserzeugenden Elemente schwer fällt, den eigenartigen Spannungsabfall bis zu einem Minimum und den darauf wieder folgenden Spannungsanstieg im Frequenzspektrum (Abb. 5) zu deuten, so läßt doch die Tatsache des parallelen Spannungsverlaufes die sichere Bestätigung der Auffassung $\mathrm{zu}$, daß es sich bei den beiden verschieden lokalisierten Ableitungen nicht um wesentlich verschiedene, sondern in der Grundfunktion gleichartige spannungserzeugende Vorgänge handeln müsse. Während die ja nicht allzugroßen Intensitätsunterschiede dieser Auffassung nicht widersprechen, verbietet sich jedoch eine gegenteilige Behauptung durch die parallele Spannungsintensität innerhalb jeder maßgeblichen Frequenz.

Gewiß, an absoluter Intensität steht die $\alpha$-Welle allen anderen weit voran. Aber auch nur bei occipitaler Ableitung ist sie dermaßen prominent; bei frontaler Ableitung, wo sie sehr an Ausprägung verliert ${ }^{11}$, braucht sie $\mathrm{z}$. B. nicht intensiver aufzutreten, als bei der Ableitung von einem Punkte des Unterschenkels! - Es wundert uns auch jetzt, wo wir z. B. vom Unterschenkel ein Frequenzspektrum aller höherfrequenten Potentialschwankungen registrieren können, das kaum von dem eines EEG unterschieden werden kann, gar nicht, daß auch (wie von so vielen Stellen des Körpers) direkt vom Gehirn selbst die gleichen Frequenzen abgeleitet werden können, wie von der darüber liegenden Kopfschwarte. Diese Coinzidenz ist zwar bedeutsam, aber weit davon entfernt, einen Beweis für die Ätiologie der von der Kopfschwarte abgeleiteten höherfrequenten bioelektrischen Erscheinungen des „Passiven EEG“ darzustellen.

Die Auffassung, daß wegen der begrenzten ,,physikalischen Streuung" auf der Kopfhaut die Möglichkeit der bioelektrisch örtlich differenzierbaren Ableitung mittels Analyse der Frequenzen über

11 E. N. Ha rvey u. G. A. H obart, J. Neurophysiol. 1, 413-430 [1938].

${ }_{12}$ A. E. K or n müll er, Die bioelektrischen Erscheinungen der Hirnrindenfelder, Thieme Leipzig 1937.
$20 \mathrm{~Hz}$ gegeben sei, ist jedenfalls nur auf intensive Hirnaktionsströme anwendbar, zu denen allerdings auch die „Krampfströme“ der Epileptiker, die Effekte direkter Hirnrindenreizung, sowie auch $\mathrm{K}$ or n mülle rs ${ }^{12}$ Feldeigenströme $\mathrm{zu}$ rechnen sind.

Ziel aller EEG-Forschung ist ja, elektrofunktionelle Äußerungen zu erforschen, die in einem direkten Zusammenhang mit Zustandsänderungen des Gehirns stehen. Die methodische Betrachtung der $\alpha$-Welle hat das große Verdienst des ersten richtunggebenden systematischen Schrittes zu diesem Ziel hin ${ }^{13}$. Mit ihrer Hilfe konnten sehr wichtige und grundlegende funktionelle Erscheinungen mit der allgemeinen Hirntätigkeit in Zusammenhang gebracht werden ${ }^{14}$. Mit dem Auftreten speziellerer Fragen, etwa der eingehenderen bioelektrischen Differenzierung der Hirnrinde, zeigten sich aber bald die Grenzen der bisherigen bewährten Methodik, indem nämlich deren Ausdrucksmöglichkeiten in keinem Verhältnis zu den Anforderungen des zu erwartenden vielfältigen Geschehens standen.

Dagegen scheint das Arbeiten mit der frequenzanalytischen Methode, welches eine unvergleichlich größere Subtilität aufweist, zur Klärung differenzierterer Probleme weitaus geeigneter zu sein. So konnte einerseits gezeigt werden, daß unter den üblichen Bedingungen des passiven EEG keine brauchbare bioelektrische Differenzierung mit der Analyse höherfrequenter Potentialschwankungen möglich sei, und deswegen auch der enkephalogene Charakter der höheren Frequenzen des passiven EEG sehr in Frage gestellt werden müsse; andererseits konnte durch eingehende Frequenzanalyse der Entstehungsort von Hirnaktionsströmen eindeutig bezeichnet und somit eine bioelektrische Differenzierung erreicht werden.

Hrn. Dr. med. et ing. W. Holzer sowie der Deut schen Forschungsgemeinschaft danke ich für die wohlwollende Förderung meiner Arbeiten.

13 W. Holzer, Physikalische Medizin in Diagnostik und Therapie, Maudrich Wien 1944, S. $89 \mathrm{ff}$.

14 vergl. dazu den systematisch bibliographischen Anhang bei A. E. K o r n müller, Klinische Elektrenkephalographie, Lehmanns Verlag München-Berlin 1944.

\section{IN MEMORIAM}

In Ergänzung zu den in Heft 1 gebrachten Todesnachrichten beklagen wir das Hinscheiden weiterer hervorragender Fachgenossen:

Johannes Abromeit, Karl von Angerer, Ludwig Diels, Gotthilf Haberlandt, Oskar Heinroth, G. Hoppe-Seyler, Martin Moebius, Friedrich Oltmanns, Wilhelm Trendelenburg, Hermann Ulich, Hans Winkler. 